\title{
The Application and Development of Artificial Blood Vessels
}

\author{
Jingwen Zhang \\ College of Materials Science and Engineering, Donghua University, Shanghai 201620, China
}

ABSTRACT: In this paper, we have summarized the types and main uses of artificial blood vessels. Also, we have analyzed the present situation of biological material-artificial blood vessels, and preliminarily explore the development.

KEYWORD: Biological materials; Artificial blood vessels

\section{INTRODUCTION}

With the development of social society, diet and other life style have changed, the number of patients with cardiovascular and arteriosclerosis is tend to go up, the aging population is increasing, more and more high technology is injected, people pay more and more attention to their own health, promoting a rapid development of biomedical material industry. Biomedical materials originated from in the 40-50's of last century and the industry was formed in the 80 's. A large number of human body transplantation such as artificial blood vessel, artificial organs, artificial esophagus, artificial kidney, artificial heart valves, artificial ligament bone, artificial joints have been successfully invented, among which artificial blood vessel prevent to be the most successful one [1].

According to the National Institute of Health, 1 in every 20 people over the age of 50 has Peripheral Vascular Disease in the United States. Many of these patients are in need of vascular prostheses that do not clog easily, prevent bloodstream infections, avoid triggering immune responses, are structurally sound, and can maintain patient for years on end. Artificial blood vessels, which are versatile and can be grown or constructed using numerous methods.

\section{ARTIFICIAL BLOOD VESSEL AND ACCESS PORT OF ARTIFICIAL BLOOD VESSEL}

As it is shown in Figure 1, an artificial blood (10) has a trunk portion (12) with both ends connected to blood vessels, and access branch portions (14), (16) and a shunt branch portion (18) branched from the trunk portion. The access branch portions (14), (16) are disposed with a solid columnshaped access port (20) that plugs these branch portions. The access port (20) has a solid center portion (22) and a tubeshaped peripheral portion (24) disposed to surround the center portion (22). The peripheral portion (24) is harder than the center portion (22) and prevents the needle from deviating from the inside of the center portion (22). This enables a puncture to be easily made in a therapy in which a puncture is highly frequently made in a blood vessel.

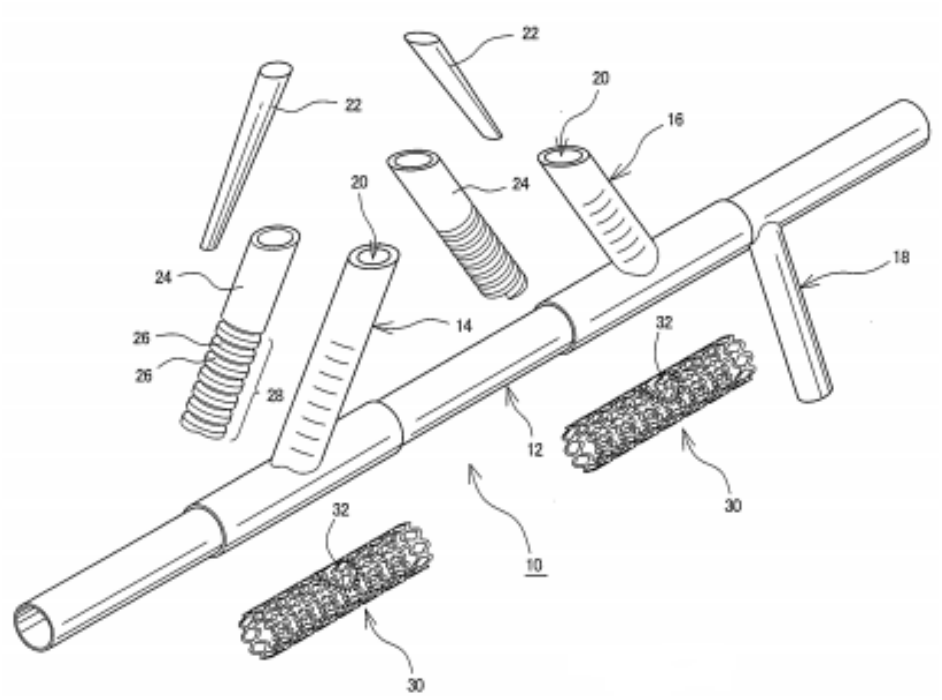

Figure 1 Artificial blood vessel and access port of artificial blood vessel 
3 THE TYPES OF ARTIFICIAL BLOOD VESSELS AND MAIN USES

With the progress of surgical procedures, the treatment of large, medium and small (and still) pulse has become a routine operation. When the body's blood vessels loss function due to disease or damage, it is necessary to carry out vascular transplantation [1]. At present the main source of vascular substitutes are biological blood vessels, artificial blood vessels and composite vessels. Biological blood vessels include autologous blood vessel, homologous blood vessel and xenogenic vascular. The latter two kinds of biological blood vessels easy to occur degeneration and strong rejection because of low patency rate, there are few clinical applications at present. Autologous blood vessels can be used as a small caliber vascular excellent substitute in the peripheral vascular reconstruction, but the source is less, the diameter and length is also restricted. Therefore, the ideal vascular substitutes clinical often needed mainly come from the artificial blood vessel and composite vessels, they have characteristics of the vascular convergence close to the human body and can maintain long-term patency and more stable performance [2, 4].

Artificial vascular are various, straight, $Y$ type and multi branch of bifurcation, with a caliber of 3$24 \mathrm{~mm}$. They can be used for the treatment of arterial diseases, venous diseases, arteriovenous fistula and other diseases, and mainly are used for the treatment of cardiovascular diseases, such as straight type is commonly used against coronary artery stenosis heart bypass surgery, a $\mathrm{Y}$ type is mainly used as implementation of abdominal aortic aneurysm endovascular exclusion, multi branch type is mainly used as heart aortic stenosis of reconstruction.

There are two methods to form artificial blood vessel: textile process and nonwoven process. Textile process mainly include organic weaving and knitting. Among all knitted artificial blood vessel, warp knitting vascular prostheses integrated the advantages of woven and knitted artificial blood vessel, becoming the one most widely used in clinical at present [2]. The nonwoven process mainly has the injection molding and the electrostatic spinning method.

No matter is the textile or the nonwoven artificial blood vessel, the properties of anti-thrombotic, antileakage and biocompatibility have not yet reached the ideal state. The main structural units of the human tissue vessel wall cells are fibrous collagen and elastin, which cause good mechanical properties and adaptability. Generally speaking, artificial vascular materials should have three basic requirements, such as lasting strength, proper pore and good compliance. The basic properties of artificial blood vessels are in order to meet the requirements of anticoagulation and anti-thrombosis. The research progress is mainly embodied in three aspects: selection of new materials, modification of blood vessels and coating, and artificial vascular endothelium.

\section{CURRENT SITUATION OF BIOLOGICAL MATERIALS FOR ARTIFICIAL BLOOD VESSELS}

From a material point of view, the artificial blood vessel is in fact a biological composite material, textile or non-woven material in which play the role of the skeleton. Artificial blood vessel has a porous structure, which can make the cell growth and cover on its surface, so as to make the vascular with biological activity [1].

Fiber used in artificial blood vessel can be natural fiber or chemical fiber, natural fibers, such as natural mulberry silk, synthetic materials in use mainly are polyester (PET), polyurethane (PU), PTFE (PTFE). The artificial blood vessels made of pan (PAN) and nylon will be degraded in human body, so these two materials have already been eliminated.

Large diameter (greater than $6 \mathrm{~mm}$ in diameter) artificial blood vessel is generally made of woven or knitted fabric, the former has better stable structure, while the elasticity the latter is better; the materials used mainly are polyester, PTFE, real silk. Small diameter (diameter less than $6 \mathrm{~mm}$ ) of artificial blood vessels are generally made of non-woven materials; the materials used are mainly PTFE and polyurethane. At present, the main problems existing in the design and application of large, from material selection to production technology, have been basically solved. Artificial blood vessels with diameter greater than $6 \mathrm{~mm}$ have been commercialized, while preparation of small caliber vascularis with diameter less than $6 \mathrm{~mm}$ is still an international problem. Diameter less than $6 \mathrm{~mm}$ for artificial blood vessel replacement arteries and veins have not received satisfactory clinical effect. The main reason lies in the thrombus formation and neointimal thickening which can cause blockages and graft failure [1, 4].

Seamless with corrugated processing artificial blood vessels are currently widely used, and the most popular is the DeBakey polyester knitted artificial blood vessels and the PTFE artificial blood vessels of Edwards.

In recent years, the research and development of artificial blood vessel have new dynamic, generally expressed in the following aspects: many researchers' studies are focused on the use of semiconductor fiber, especially in absorbable bicomponent fiber, surface modification and applications of three dimensional scaffold, mainly four types: cavity surface lined with endothelial cells of the vascular prostheses, autologous tissue graft type artificial blood vessel, wall composite artificial blood vessel, the sur- 
face has anti thrombosis in artificial blood vessel [6, 7].

\section{THE DEVELOPMENT OF ARTIFICIAL BLOOD VESSELS IN CHINA}

In our country, the artificial blood vessel research starts not late, all of the produced real silk, polyester woven and dacron vascular graft had clinical application, but the study of artificial blood vessels is far behind the developed countries [4]. At present, the market is still mainly imported artificial blood vessels. In 1963, the "polyester artificial blood vessels" (straight type and Y type) was developed successfully, and was officially used in the human body. A series of technical indicators like elasticity, strength, and bleeding and tube wall thickness of artificial blood vessel have reached the requirements of the human body, known as "China's first generation of artificial blood vessel". In September of 1979, clinical application of the "woven polyester Plush type artificial blood vessel" obtained success. As it was identified by authority of experts in medical, the artificial blood vessel had unique pipe wall structure and excellent biomedical properties, regardless of the elasticity, strength, or in bleeding, easy sewing, healing degree were better than the first generation artificial blood vessel. Therefore, it is known as China's "second generation artificial vessels"[5].

There are three main factors limiting the development of artificial blood vessels in our country. One is the limitation of development of polymer materials, such as the development of polyurethane products for small diameter artificial blood vessel, the development of good biological properties degradable materials for vascular tissue engineering substrates is not satisfactory. Second is the lack of good cooperation between the various industries, the research and development of artificial blood vessel is an interdisciplinary disciplines involves materials engineering, biological engineering, medicine and textile engineering, only by breaking the gap between each specialized industry, close cooperation, broad horizons, can we achieve greater progress. Thirdly, the lack of study on the characterization of biomechanical properties of artificial blood vessel, there is no suitable test methods of product quality inspection and evaluation of in vitro in the research and development process, only can through short term animal tests. On one hand, it improved the experimental funding and the development cycle. On the other hand, the performance of the developed products cannot be given the test targets in conformity with international standards, which could be trusted to consumers, limiting the development of products in the market, resulting in the situation that imported artificial vascular products almost have been a monopoly in China's market today. Therefore, we must fully understand the gap between domestic and foreign research of artificial blood vessels and the restriction of the development for artificial vascular, as soon as possible to achieve localization of series products [4].

\section{THE RESEARCH AND EXPLORATION OF ARTIFICIAL BLOOD VESSELS}

Donghua University have continued in-depth study and success of the test for the preparation technology of artificial blood vessels in the bifurcation [8], the double layer of woven artificial vascular [9], multi-layer woven artificial vascular [10]. The textile type artificial blood vessel existed is made by weaving or knitting method, and all the blood vessel walls are a single layer structure. To avoid the capillary hemorrhage of tube wall, the commonly used method is to increase the tightness of woven fabric or to change the surface structure of the wall tube of artificial blood vessel, however, the above methods are not conducive to the proliferation of human cells and tissues after the surgery; and the coagulation pretreatment will increase the complexity of operation; coating may cause an allergic reaction, so the all the methods are not ideal. Ding Xin found that artificial blood vessels with two layers of different woven structures can prevent the operation of the blood at the same time the smooth proliferation of cell tissue. This kind of specially designed tubular fabric can be used as an artificial blood vessel for surgery without coating and pre coagulation, so it has broad application prospects. But the preparation technology of this kind of multi-layer woven artificial blood vessel is still blank, because the effective design principle and the preparation method of this kind of tubular woven fabric have not been found yet [10].

The relationship between the characteristics of the hydraulic performance of the textile based artificial blood vessel wall was studied by Donghua University. Although the porosity of the artificial vascular fabric affects its water permeability to a large extent, the water permeability of the fabric is very large when the fabric structure is not the same even if the porosity is close. And for the general structure of artificial vascular fabric, its average pore size and water permeability have a good linear correlation [11]. The preparation and application of amphiphilic polyurethane were studied in Anhui University. Amphiphilic polyurethane material has good biological compatibility, blood compatibility, and is not absorbed by the body protein, its performance can be conveniently adjusted according to the requirements, it can be used for the production of artificial blood vessel, artificial skin, cartilage and cardiac valve biomaterial, and it has got wide attention by the medical community [12]. 
As it is reported, Japan has successfully developed silk artificial blood vessels and got bulk production technology patents, filling the gaps in this field all over the world. The manufacture of pure silk prosthesis use special processed silk, with unique knitting technology rolled into a cylindrical and the outside with alternative silk as a protective coating, to prevent the leakage of blood, which can be said to be the best combination of silk and knitting technology [13].

\section{SUMMARY}

The main focus of the future research for artificial blood vessels: the technology to solve the patency rate of small caliber artificial blood vessels. Artificial blood vessel completely replace vessels may be the dream of mankind, but with the discovery of new materials and further understanding on human vascular biology, and efforts through the tissue engineering and gene engineering, human will be more and more near from this dream [3].

\section{REFERENCES}

[1] Lihua Zong, Xiangyi Ji. The application of nonwoven materials for surgical implants[J]. Nonwovens, 2004, 9, 12(3).

[2] Rui Li. Structural characteristics of artificial blood vessels of different textile bases[J]. Progress in Textile Science and Technology, 2007, 6.

[3] Jiwei Zhang. Performance requirements and research status of artificial blood vessel[J]. Chinese Journal of Practical Surgery, 2007, 7, 27(7).

[4] Lixia Jia, Lu Wang, Kai Lin. The developing course and direction of artificial blood vessel[J]. Shanghai Textile Science and Technology, 2003, 6, 31(3).

[5] Xiaoping Qian. The research and development of the first and second generation of artificial blood vessels in China[J]. Silk, 2011, 9, 9.

[6] Xiangbo Sun, Qingshan Li, Guirong Pen. The research and development of artificial blood vessels[J]. The eighth Symposium on Ffunctional Textiles and Nanotechnology, 2008,8,28.

[7] Jianyong Feng. The development and prospect of biomedical textiles for biological transplantation[J]. Topnew Cup Ninth Functional Textiles and Nanotechnology Research Symposium, 2009.

[8] Gang Li, Yuling Li, Xuwei Chen, et al. The study on the technology of artificial blood vessels[J].Industrial Textiles, 2008,8.

[9] Sheng Wang, Yuling Li, Gang Li, et al.Preparation of double layer cross woven artificial blood vessel[J]. Textile Journal, 2009, 5, 30(5).

[10] Gang Li, Yuling Li, Xuwei Chen, et al. Design and fabrication of multilayer woven artificial blood vessels[J]. Journal of Donghua University, 2009, 6, 35(3).

[11] Lixia Jia, Lu Wang, Kai Ling. Water permeability and pore size distribution of artificial blood vessel wall in textile base[J]. Cotton Textile Technology, 2009, 9, 37(9).
[12] Huiqing $\mathrm{Hu}$, Jianjun Yang, Qingyun $\mathrm{Wu}$, et al. Preparation and application of amphiphilic polyurethane[J]. Polyester Industry, 2008, 5, 21(3).

[13] Williams,S.K., D.G. Rose, et al. Microvascular endothelial cell sodding of ePTFE vascular grafts: improved patency and stability of the cellular lining[J]. Journal of Biomedical Materials Research, 1994, 28: 203-212. 